\title{
Stereotactic body radiotherapy based treatment for hepatocellular carcinoma with extensive portal vein tumor thrombosis
}

Yongjie Shui ${ }^{1}$, Wei $\mathrm{Yu}^{1,4}$, Xiaoqiu Ren ${ }^{1,4}$, Yinglu Guo ${ }^{1,4}$, Jing X $\mathrm{u}^{1,4}$, Tao $\mathrm{Ma}^{2}$, Bicheng Zhang ${ }^{1}$, Jianjun Wu ${ }^{3}$, Qinghai Li ${ }^{3}$, Qiongge Hu' ${ }^{1}$ Li Shen ${ }^{1}$, Xueli Bai ${ }^{2}$, Tingbo Liang ${ }^{2}$ and Qichun Wei ${ }^{1,4^{*}}$

\begin{abstract}
Background: There is currently no worldwide consensus for the management of hepatocellular carcinoma (HCC) with portal vein tumor thrombus (PVTT). We evaluated the efficacy of stereotactic body radiotherapy (SBRT) as the initial treatment for HCC with extensive PVTT based on a relatively large number of patients.

Methods: In our multidisciplinary approach for patients with hepatobiliary tumors, SBRT is recommended for unresectable HCC with PVIT or those with contraindication for transarterial chemoembolization (TACE). The aim is to shrink the tumor thrombus and preserve adequate portal venous flow, thus facilitating subsequent treatments such as TACE and tumor resection. In the present study, 70 continuous cases of HCC patients with extensive PVIT initially treated with SBRT were studied. The median follow-up period was 9.5 months (range, 1.0-21.0 months). The dynamic changes of tumor thrombosis with time after SBRT were also analyzed.

Results: The median survival time for the whole group was 10.0 months ( $95 \% \mathrm{Cl}, 7.7-12.3$ months), with a 6- and 12month overall survival (OS) rate of $67.3 \%$, and $40.0 \%$ respectively. Patients who received combined SBRT and TACE showed significantly longer OS than those without indication for TACE after SBRT (12.0 \pm 1.6 vs. $3.0 \pm 1$. 0 months). Patients with good response to radiation usually had better survival. SBRT was well tolerated in our patient series.
\end{abstract}

Conclusions: In conclusion, SBRT used as the initial treatment for HCC patients with extensive PVIT originally unsuitable for resection or TACE can achieve adequate thrombus shrinkage and portal vein flow restoration in the majority of cases. It could thus offer the patients an opportunity to undergo further treatment such as resection or TACE procedure. Such therapeutic strategy may result in survival advantage, especially for those who do receive combined modality with SBRT.

Keywords: Hepatocellular carcinoma, Portal vein tumor thrombosis, Stereotactic body radiotherapy, Transarterial chemoembolization

\footnotetext{
*Correspondence: Qichun_Wei@zju.edu.cn

This work has been accepted for a "ePoster presentation" at the 13th IHPBA World Congress taking place in Geneva, Switzerland, 4-7 September 2018 (presentation code "EP01B-015")

'Department of Radiation Oncology, The Second Affiliated Hospital, Zhejiang University School of Medicine, Jiefang Road 88, Hangzhou 310009, People's Republic of China

${ }^{4}$ Ministry of Education Key Laboratory of Cancer Prevention and Intervention, Zhejiang University Cancer Institute, Hangzhou 310009, People's Republic of China

Full list of author information is available at the end of the article
}

(c) The Author(s). 2018 Open Access This article is distributed under the terms of the Creative Commons Attribution 4.0 International License (http://creativecommons.org/licenses/by/4.0/), which permits unrestricted use, distribution, and reproduction in any medium, provided you give appropriate credit to the original author(s) and the source, provide a link to the Creative Commons license, and indicate if changes were made. The Creative Commons Public Domain Dedication waiver (http://creativecommons.org/publicdomain/zero/1.0/) applies to the data made available in this article, unless otherwise stated. 


\section{Background}

Hepatocellular carcinoma (HCC) is the sixth most prevalent cancer worldwide [1]. In China, $\mathrm{HCC}$ is the fourth most commonly diagnosed cancer and the third leading cause of cancer death [2]. Macrovascular invasion (MVI) is common in HCC; in such case, tumor cells invade the portal veins, hepatic veins, or the inferior vena cava in the liver [3, 4]. Portal vein tumor thrombus (PVTT) is the most common form of MVI in $\mathrm{HCC}$, with an incidence ranging from 44 to $62.2 \%$ [5]. About $10 \%$ to $60 \%$ of HCC patients have PVTT at the time of diagnosis $[6,7]$. Although the survival rate of patients with $\mathrm{HCC}$ has been improved recently, the prognosis for those with PVTT remains poor, as their median survival is only 2-4 months via supportive care [8]. Overall, PVTT plays a major role in predicting the therapeutic outcome and clinical staging of HCC $[9,10]$.

There is currently no widely-accepted consensus for the management of HCC with PVTT. According to some guidelines in Europe and America, HCC with PVTT is regarded as Stage $\mathrm{C}$ per Barcelona Clinic Liver Cancer (BCLC) Staging system, and sorafenib alone is recommended as the treatment of choice [11]. In Southeast Asian countries, modalities including surgery, radiotherapy, transarterial chemoembolization (TACE), and/or sorafenib are involved in the multidisciplinary treatment of HCC with PVTT $[12,13]$. Surgical treatment is recommended for suitable HCC patients with type I/II PVTT [14-16]. However, the management of HCC with extensive portal vein involvement remains complicated and controversial. Patients with PVTT extended to the main portal vein or the contralateral branch had no survival benefit after surgical treatment [17]. TACE has been contraindicated in the treatment of HCC patients with PVTT involving the main trunk and/or first branch of the portal vein due to potential risk of liver infarction and hepatic failure resulting from ischemia $[6,18]$. Several recent reports have shown that selected patients with PVTT may benefit from more aggressive treatment modalities $[6,19]$ such as a combination of radiotherapy and TACE. So far, few studies have investigated the efficacy of stereotactic body radiotherapy (SBRT) for the treatment of PVTT [20-22].

SBRT has emerged as a new technology which delivers large dose of radiation to a target in a few fractions. By taking advantage of the technologic advancements in precise radiation dose delivery, respiratory motion management, and daily image guidance, SBRT enables accurate targeting of multiple high-dose radiation beams to treat a tumor volume, typically over 1 to 5 fractions. Characterized by rendering a higher biologically effective dose (BED) than conventionally fractionated radiotherapy, minimal invasiveness and decreased morbidity, SBRT can be finished in a week. Such relatively short treatment course may post less interference with other therapeutic measures which may also benefit the patient sequentially in time.

In our multidisciplinary management for patients with hepatobiliary tumors, SBRT is recommended for patients with unresectable HCC with PVTT and those with contraindication for TACE. The aim is to shrink the tumor thrombus and preserve adequate portal venous flow, thus facilitating subsequent treatments such as TACE or tumor resection. In the present report, 70 continuous HCC with extensive PVTT initially treated with SBRT were analyzed. The therapeutic response, survival, safety, and treatment strategy were discussed.

\section{Methods}

Patient population and radiation treatment

This retrospective study was performed with the approval of our local ethics committee. From December 2015 to June 2017, 70 continuous HCC patients with PVTT received SBRT at the Second Affiliated Hospital, Zhejiang University School of Medicine. The diagnosis of HCC was based on the American Association for the Study of Liver Disease (AASLD) guideline [23]. Portal vein invasion was identified by the presence of a low-attenuation intraluminal filling defect adjacent to the primary tumor as discerned from contrast-enhanced computed tomography (CT). Cheng's classification of PVTT was applied in this study, and comprised of four levels based on the extent of tumor thrombus in the portal vein (Fig. 1): type $\mathrm{I}_{0}$, tumor thrombus found only under microscopy; type I, tumor thrombus involving segmental or sectoral branches of the portal vein or above; type II, tumor thrombus involving the right/left portal vein; type III, tumor thrombus involving the main portal vein; and type IV, tumor thrombus involving the superior mesenteric vein [24]. Basic criteria for SBRT: (1) tumor thrombus involving the main trunk and/or first branches of the portal vein, unsuitable for surgery or TACE; (2) an Eastern Cooperative Oncology Group (ECOG) performance status (PS) of $0-2$; (3) no refractory ascites; (4) Child-Pugh class $\mathrm{A}$ and $\mathrm{B}$, or class $\mathrm{C}$ with good performance status; (5) no previous radiotherapy to the liver; and (6) more than $700 \mathrm{cc}$ of uninvolved liver. A preliminary estimate of the uninvolved liver volume was made using 3D imaging software (IQQA-Liver, EDDA Technology Inc., Princeton, NJ, USA), whereas its exact volume was verified from the subsequent SBRT planning dosimetry (Eclipse software, Varian ${ }^{\oplus}$ Medical Systems, Palo Alto, CA).

All patients were immobilized in a stereotactic body frame (Karity, Guangzhou, China) with customized vacuum cushion and abdominal compression for control of respiratory motion. Oral contrast agent $(50 \mathrm{ml}$ of $3 \%$ 

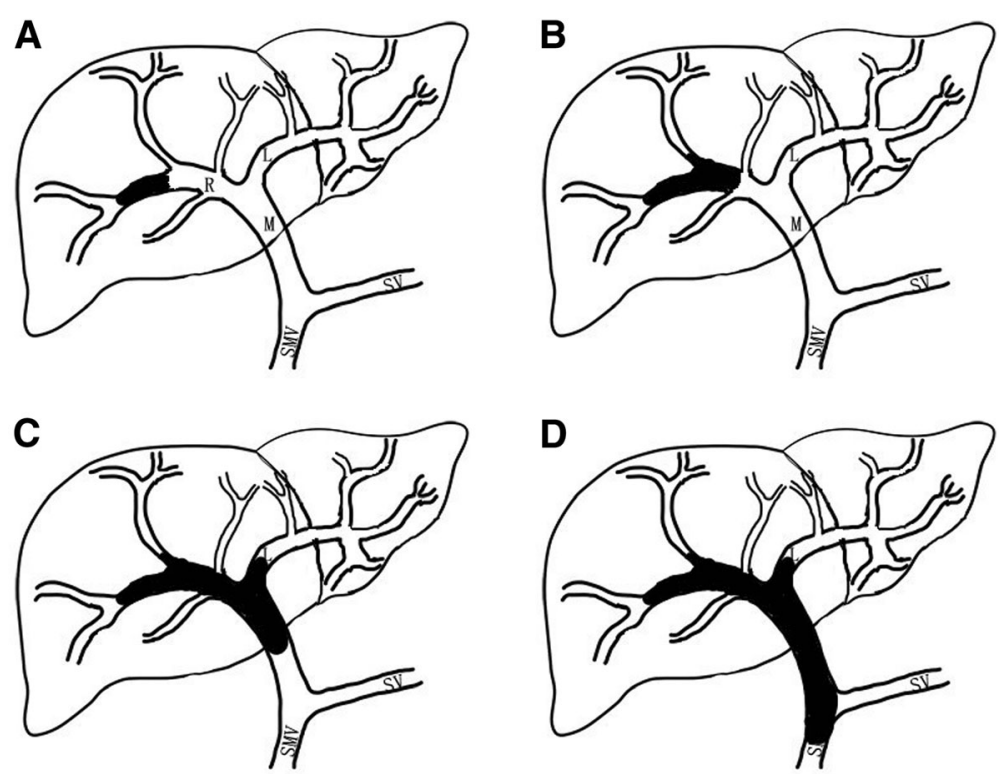

Fig. 1 Cheng's classification of hepatocellular carcinoma with portal vein tumor thrombus. a type l, tumor thrombus involving segmental or sectoral branches of the portal vein or above; $\mathbf{b}$ type II, tumor thrombus involving the right/left portal vein; c type III, tumor thrombus involving the main portal vein; d type IV, tumor thrombus involving the superior mesenteric vein

Ioversol) was administered before CT simulation. Four-dimensional contrast-enhanced computed tomography (4DCT) simulation (Light Speed RT, GE) was performed at 2.5-mm slice thickness. For breathing movement amplitude too small to be detected during 4DCT scanning, scans were taken at end-expiration phase, end-inspiration phase, and during quiet free-breathing under abdominal compression. The CT scan during free breathing was then used for treatment planning. The gross tumor volume (GTV) represented the tumor thrombosis visualized on the contrast enhanced CT, and magnetic resonance (MR) images. If the primary hepatic lesion was small (less than $5 \mathrm{~cm}$ ) and adjacent to the PVTT, both portal vein tumor thrombus and the primary lesion were contoured as the GTV. Internal target volume (ITV) was defined as the volumetric sum of GTVs in the multiple phases. The planning target volume (PTV) included ITV with 3-5 mm margins, to account for daily set-up variations. PTV was adjusted manually to minimize overlapping the gastrointestinal tract when indicated. The mean volume of PTV was $390.8 \pm 37.6 \mathrm{~cm}^{3}$, varying widely from a minimum of $63.0 \mathrm{~cm}^{3}$ to a maximum of $1452.9 \mathrm{~cm}^{3}$. Plans were devised such that the prescription dose was prescribed at the isodose line encompassing $>95 \%$ of the PTV. The preferred maximum dose within the PTV was between 110 and $130 \%$ of the prescribed dose. The median prescription dose to PTV was 40 Gy (range, 25-50) in five fractions administered over a week. Organs at risk (OARs) included liver, kidneys, stomach, duodenum, small intestine, colon and spinal cord. Dose-volume planning objectives for the OARs were defined as follows
(Table 1): normal liver, mean dose $\leq 15$ Gy; bilateral kidney, mean dose $\leq 12 \mathrm{~Gy}$; and spinal cord, maximal dose $<27$ Gy. The maximal dose to $1 \mathrm{cc}$ (D1cc) was limited to $31 \mathrm{~Gy}$ for the gastrointestinal tract including stomach, duodenum, small intestine and colon. Normal liver volume was defined as the total liver volume minus the PTV. Target and OAR contouring were performed using Varian Dosimetrist and Oncologist software (Eclipse software, Varian $^{\bullet}$ Medical Systems, Palo Alto, CA). Treatment was delivered with a Varian ${ }^{\bullet}$ Trilogy $^{\mathrm{Tw}}$ linear accelerator (Varian ${ }^{\circ}$ Medical Systems, Palo Alto, CA) using X-ray beams of 6-10 MV energy. Daily image guidance was performed by means of kilovoltage cone-beam CT, with

Table 1 Dose-volume constraints to organs at risk

\begin{tabular}{ll}
\hline Organs at risk & Constraints (5 Fractions) \\
\hline Liver & mean dose $\leq 15 \mathrm{~Gy},>700 \mathrm{cc}$ \\
Kidney & mean dose $\leq 12 \mathrm{~Gy}$ \\
& $\mathrm{~V} 18<33 \%$ \\
Spinal cord & maximal dose $<27 \mathrm{~Gy}$ \\
Stomach & $\mathrm{V} 31<1 \mathrm{cc}$ \\
& $\mathrm{V} 20<3 \mathrm{cc}$ \\
Duodenum & $\mathrm{V} 31<1 \mathrm{cc}$ \\
& $\mathrm{V} 20<3 \mathrm{cc}$ \\
Small intestine & $\mathrm{V} 31<1 \mathrm{cc}$ \\
& $\mathrm{V} 20<3 \mathrm{cc}$ \\
Colon & $\mathrm{V} 31<1 \mathrm{cc}$ \\
& $\mathrm{V} 20<10 \mathrm{cc}$
\end{tabular}

Abbreviations: $\mathrm{Vxx}=$ the volume or percentage of organ receiving more than the $x x$ Gy 
patients' $3 \mathrm{D}$ positioning verified prior to each radiation treatment session.

\section{Evaluation}

Patients were assessed weekly for toxicities in the first month after SBRT, monthly for the following two months, and once every three months thereafter. Treatment-associated acute and late toxicities were scored according to the Common Terminology Criteria for Adverse Events (CTCAE; version 3.0). Tumor response was assessed using the modified Response Evaluation Criteria in Solid Tumors (mRECIST) criteria [25]. The response of PVTT to SBRT was evaluated by dynamic contrast enhanced CT and/or MRI at 1, 3, and then every 3 months after SBRT. Biochemical response was assessed in patients with elevated alpha-fetoprotein (AFP) level before radiotherapy and defined as either $\mathrm{a} \geq 50 \%$ reduction or normalization of the AFP level within one month after SBRT.

\section{Follow-up and statistical analysis}

The cutoff date for the last follow-up was February 28, 2018, for the censored data analysis. The median follow-up period was 9.5 months (range: 1.0-21.0). The overall survival (OS) was calculated from the start of SBRT to the date of either death or the last follow-up visit. The Kaplan-Meier method was used to analyze the OS, with log-rank test used to examine group differences. Cox regression model was used for multivariate analysis. All statistical analyses were performed using SPSS software package (version 20.0; SPSS Inc., Chicago). A $p$-value of $<0.05$ was considered statistically significant.

\section{Results}

A total of $70 \mathrm{HCC}$ patients with PVTT were irradiated and included in this analysis. Table 2 shows the patients' characteristics, classification of PVTT and radiotherapy scheme. The median age at diagnosis of PVTT was 53.8 years (range: $25-75$ ). The median time from primary HCC diagnosis to SBRT treatment was 7 months (range: $0-85$ ). The median time interval between diagnosis of PVTT and SBRT treatment was 1.2 months (range $0-12$ ). Tumor thrombosis involving the first order portal vein branches without main portal vein involvement (TypeII) was found in 42 patients (60\%). Twenty- seven patients $(38.6 \%)$ had tumor thrombosis invading the main trunk (Type III), one patient $(1.4 \%)$ had tumor thrombosis invaded the superior mesenteric vein, portal vein main trunk and both first branches (Type IV).

Twenty patients $(28.6 \%)$ received SBRT alone. Another 46 (65.7\%) patients received TACE (1-5 cycles, median 2.4 cycles) after SBRT; among them, 3 underwent further radioactive seeds implantation, and one received
Table 2 Patient characteristics

\begin{tabular}{|c|c|}
\hline Characteristics & n (\%) \\
\hline \multicolumn{2}{|l|}{ Age, y } \\
\hline$\geq 50$ & $48(68.6)$ \\
\hline$<50$ & $22(31.4)$ \\
\hline \multicolumn{2}{|l|}{ Gender } \\
\hline Male & $59(84.3)$ \\
\hline Female & $11(15.7)$ \\
\hline \multicolumn{2}{|c|}{ Therapeutic modalities } \\
\hline SBRT alone & $20(28.6)$ \\
\hline SBRT+TACE & $46(65.7)$ \\
\hline SBRT+Surgery & $4(5.7)$ \\
\hline \multicolumn{2}{|l|}{ Dose, Gy } \\
\hline$\leq 35.0$ & $29(41.4)$ \\
\hline$\geq 40.0$ & $41(58.6)$ \\
\hline \multicolumn{2}{|l|}{ Types of PVTT } \\
\hline$\|$ & $42(60.0)$ \\
\hline III & $27(38.6)$ \\
\hline IV & $1(1.4)$ \\
\hline \multicolumn{2}{|l|}{$\mathrm{HBsAg}$} \\
\hline Negative & $12(17.1)$ \\
\hline Positive & $58(82.9)$ \\
\hline \multicolumn{2}{|c|}{ Child-Pugh classification } \\
\hline A & $45(64.3)$ \\
\hline B & $24(34.3)$ \\
\hline C & $1(1.4)$ \\
\hline \multicolumn{2}{|l|}{ PS (ECOG) } \\
\hline 0 & $56(80.0)$ \\
\hline 1 & $14(20.0)$ \\
\hline \multicolumn{2}{|c|}{ Origination of PVTT } \\
\hline Right branch & $49(70.0)$ \\
\hline Left branch & $21(30.0)$ \\
\hline \multicolumn{2}{|l|}{ AFP, ng/L } \\
\hline$\leq 20$ & $13(18.6)$ \\
\hline $21 \sim 399$ & $17(24.3)$ \\
\hline$\geq 400$ & $40(57.1)$ \\
\hline \multicolumn{2}{|l|}{$\mathrm{PLT}, 10^{9} / \mathrm{L}$} \\
\hline$\geq 100$ & $39(55.7)$ \\
\hline$<100$ & $31(44.3)$ \\
\hline \multicolumn{2}{|l|}{$\mathrm{HGB}, \mathrm{g} / \mathrm{L}$} \\
\hline$\geq 120$ & $42(60.0)$ \\
\hline$<120$ & $28(40.0)$ \\
\hline \multicolumn{2}{|l|}{ TBIL, $\mu \mathrm{mol} / \mathrm{L}$} \\
\hline$\geq 20$ & $34(48.6)$ \\
\hline$<20$ & $36(51.4)$ \\
\hline
\end{tabular}

Albumin, $g / L$ 
Table 2 Patient characteristics (Continued)

\begin{tabular}{ll}
\hline Characteristics & $\mathrm{n}(\%)$ \\
\hline$\geq 35$ & $41(58.6)$ \\
$<35$ & $29(41.4)$ \\
ALT, U/L & \\
$\geq 50$ & $25(35.7)$ \\
$<50$ & $45(64.3)$ \\
AST, U/L & \\
$\geq 50$ & $48(68.6)$ \\
$<50$ & $22(31.4)$ \\
\hline
\end{tabular}

Abbreviations: SBRT Stereotactic body radiotherapy, Types types of tumor thrombi, HBsAg Hepatitis B surface antigen, PS Performance status, ECOG Eastern Cooperative Oncology Group, AFP Alpha-fetoprotein, PLT Platelet, HGB Hemoglobin, TBIL Total bilirubin, ALT Alanine aminotransferase, AST Aspartate aminotransferase

radiofrequency ablation (RFA) after TACE. The final 4 (5.7\%) patients underwent surgery, including 3 with respective liver lobectomy at 3, 8 and 10 weeks after SBRT, and one patient underwent liver transplantation 4 weeks after SBRT.

\section{PVTT response}

Fifty-three cases had contrast-enhanced CT/MRI around 4 weeks after SBRT, partial response (PR), stable disease (SD), and progression disease (PD) were observed in 41 (77.4\%), 8 (15.1\%), and $4(7.5 \%)$, respectively. While none had achieved complete response (CR) by this time point, there were 5 patients (9.4\%) reached near CR with minimal residual disease less than $10 \%$ of original enhanced size. Local control (LC) (inclusive of partial and stable response) was thus achieved in $92.5 \%$ of the treated lesions.

At 3 months after SBRT, 62 patients were assessed for PVTT therapeutic responses. CR, PR, and SD were observed in $6(9.7 \%), 43(69.4 \%)$, and $4(6.4 \%)$ of the patients, respectively. LC (inclusive of CR, PR and SD) was achieved in $85.5 \%$ of the treated lesions. The rest 9 patients (14.5\%) had PD, including 3 cases having developed PD at the first follow-up.

Six months after SBRT, assessments of PVTT therapeutic response were done in 31 patients, $C R, P R, S D$, and PD were observed in 10 (32.2\%), 16 (51.6\%), 2 (6.4\%), and 3 (9.8\%) (including 1 case evaluated as PD beforehand) patients, respectively. Sixteen patients had follow-up imaging around 9 months after SBRT, and the respective CR and PR rate were $56.25 \%$ (9 patients) and $43.75 \%$ (7), with the corresponding figures at 12 months being $66.7 \%$ (10) and $33.3 \%(5)$.

Of the 57 patients with elevated AFP levels before SBRT, 29 patients $(54.7 \%)$ exhibited $\geq 50 \%$ reduction in the AFP levels within one month after SBRT, with 6 cases $(11.3 \%)$ had AFP in the normal range.

\section{Overall survival}

With median follow-up period of 9.5 months, 25 patients $(35.7 \%)$ were still alive at the time of the current analysis. The median survival time for the whole group was 10.0 months (95\% CI, 7.7-12.3), with 6and 12 -month OS rates of $67.3 \%$, and $40.0 \%$, respectively (Fig. 2a).

Median survival times were $12.0 \pm 1.6$ and $3 \pm$ 1.0 months for those receiving TACE after SBRT and SBRT alone, respectively. Patients who received combined treatments of SBRT and TACE showed significantly longer OS than those unable to undergo TACE after SBRT $(p<0.001$; Fig. 2b). The 6- and 12-month OS rates were $82.8 \%$, and $49.7 \%$ for patients receiving SBRT plus TACE, and $30.0 \%$, and $10.0 \%$ for those receiving SBRT alone. Four patients underwent tumor resection after SBRT, with 1 patient died at the fourth month while the other 3 were alive at their respective follow-up time of 13, 15 and 15 months.

The median survival time for patients with PR, SD and PD of the PVTT at 3 months after completion of SBRT was 13.0, 8.0 and 4.0 months respectively. The median survival for the 6 patients with CR was deficiency because only 2 patients died at seventh and eleventh month. The other 4 patients remained alive, with 1 patient assessed at 15-month and 3 at 16-month follow-up points after SBRT. The median survival times for patients with LC (inclusive of CR, PR and SD) of the PVTT were better than those with PD after SBRT (13.0 vs. 4.0 months, $p<0.001$ ) (Fig. 2c).

Interestingly, patients with PVTT at the left branch location seemed to have longer median OS than those with thrombosis in the right branch of portal vein (13.0 vs. 8.0 months, $P=0.079$ ) (Fig. $2 \mathrm{~d}$, Table 3 ).

\section{Toxicity}

Patients tolerated the SBRT generally well, since a very mild pattern of acute toxicity was observed. No treatment-related deaths or serious adverse events were seen within 3 months after SBRT. Acute radiation side effects were mild, including nausea, vomiting, anorexia, and abdominal pain in some cases. Grade-3 leukopenia and thrombocytopenia were seen in $5(7.1 \%)$ and $4(5.7 \%)$ patients, respectively. Grade-3 liver enzyme and bilirubin elevation were found in 3 $(4.3 \%)$ and $8(8.6 \%)$ cases, while grade- 3 albumin decrease was seen in 11 (15.7\%). No grade-4 hematologic toxicity, liver enzyme, bilirubin and albumin level change was seen. No radiation induced liver disease was encountered in the entire patient group. Later toxicities such as gastrointestinal stenosis, bleeding, perforation and ulcer were not observed during the follow-up periods. 

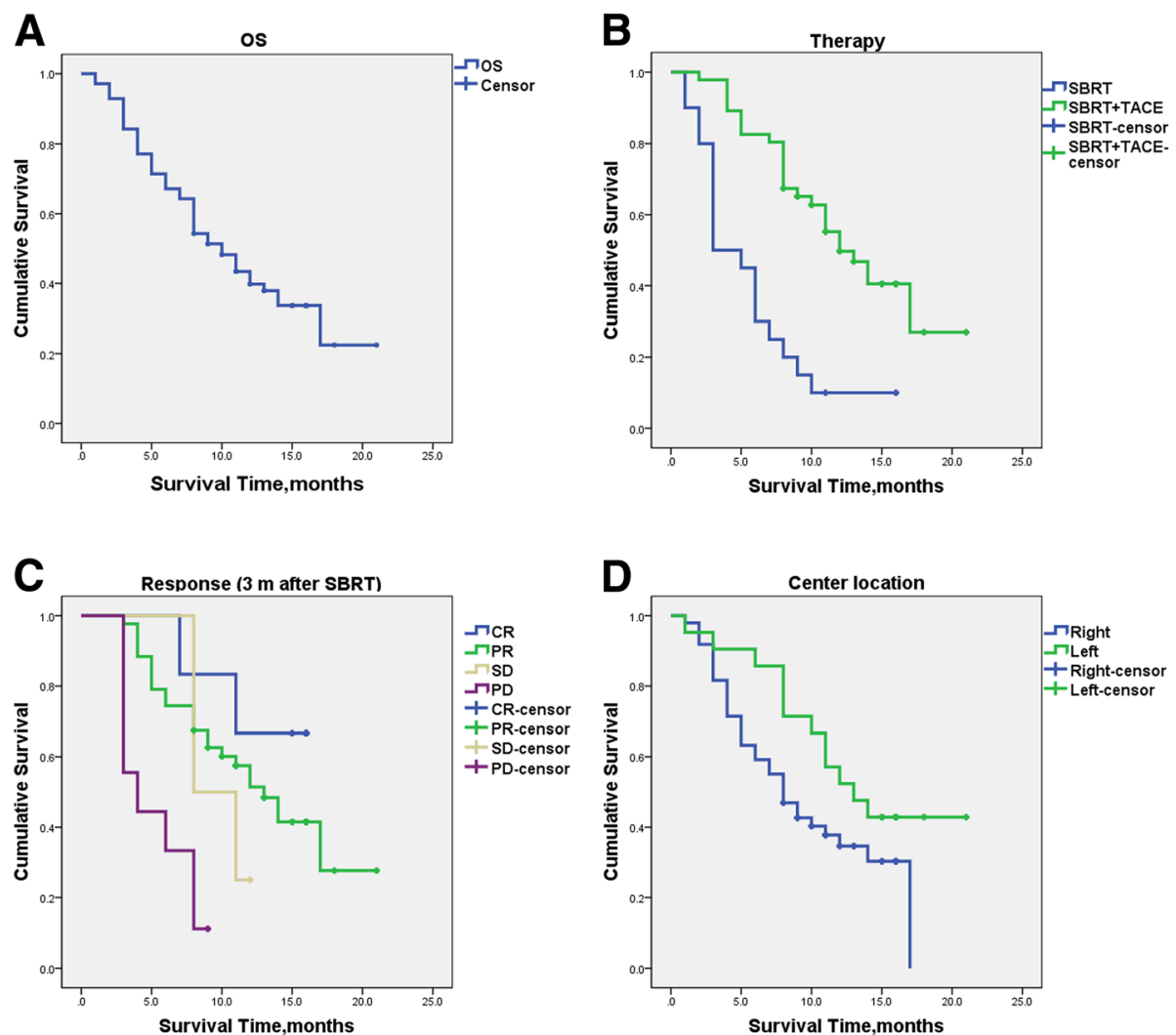

Fig. 2 Overall survival of the whole group and subgroup. a Overall survival curve for the whole group of 70 HCC patients with PVTT; b Overall survival curves according to the additional therapy; c Overall survival curves according to the response at three months after completion of SBRT; d Overall survival curves according to the center location of PVTT

Only one patient $(1.4 \%)$ showed progression of ChildPugh class from A to $C$ within 3 months after SBRT, while progression from class $\mathrm{B}$ to $\mathrm{C}$ was observed in 2 cases. All these 3 patients were deteriorated due to intrahepatic tumor progression. Downgrading of Child-Pugh class from $B$ to $A$ was observed in 3 cases.

\section{Discussion}

In the present study, SBRT was used as the first therapy for the management of HCC patients with extensive PVTT. These patients were originally unsuitable for surgery or had contraindication for TACE. SBRT induced prominent response in the PVTT. The objective response rate at $1,3,6$ months were $77.4 \%, 79.1 \%$ and $83.8 \%$, respectively. Within 1 month after SBRT, 5 cases reached near $\mathrm{CR}$, i.e., minimal residual disease less than $10 \%$ of initial lesion. Remarkably, the CR rate increased with time, from $9.7 \%$ at 3 months to $32.2 \%$ at 6 months after SBRT. For the 16 patients with imaging reassessment at 9 months after treatment, more than half achieved CR. Progression after SBRT was found in about $17.1 \%$ of the cases, with most PD occuring within the first 3 months. In our series, 4 patients had PD of the
PVTT within 1 month, 6 between 1 and 3 months, and only 2 patients at 6 months. To the best of our knowledge, this appears to be the first report describing the dynamic changes of tumor thrombosis after SBRT.

Around $70 \%$ of our PVTT cases shrank to a degree of PR in the first few weeks. Most achieved restoration of portal vein flow, which relieved the associated symptoms and made it feasible for patients to endure further interventional therapies. The strategy seems practical to shrink the thrombosis and restore the portal flow by SBRT, thus enable the patients to have the opportunity to receive subsequent treatments, such as tumor resection or TACE procedure.

The median survival time from the start of SBRT for this patient series was 10.0 months, with the respective 6- and 12 -month OS rates of $67.3 \%$ and $40.0 \%$. Published results concerning SBRT in the treatment of PVTT are scarce, and median survival time has been reported to vary from 8 to 13 months, with 1-year OS rate from 43.2 to $50.3 \%$ [22, 26, 27]. In a prospective study of phase I and II trials reported by Bujold et al. [28], 56 HCC patients with tumor thrombosis showed a 1-year OS rate of $44 \%$ after SBRT, with which our result seems 
Table 3 Analysis of prognostic factors for survival

\begin{tabular}{|c|c|c|c|}
\hline & $\mathrm{N}$ & Median survival (month; 95\% Cl) & $p$ values \\
\hline \multicolumn{3}{|l|}{ Gender } & 0.13 \\
\hline Male & 59 & $11.0(7.6-14.4)$ & \\
\hline Female & 11 & $8.0(1.5-14.5)$ & \\
\hline \multicolumn{3}{|l|}{ Age, y } & 0.033 \\
\hline$<50$ & 22 & $17.0(17.0-17.0)$ & \\
\hline$\geq 50$ & 48 & $8.0(6.3-9.7)$ & \\
\hline \multicolumn{3}{|l|}{ AFP, ng/L } & 0.438 \\
\hline$\leq 20$ & 14 & $8.0(2.9-13.1)$ & \\
\hline $21-399$ & 18 & $14.0(11.9-16.1)$ & \\
\hline$\geq 400$ & 38 & $8.0(5.6-10.4)$ & \\
\hline \multicolumn{3}{|c|}{ Additional treatment after RT } & 0.00 \\
\hline SBRT alone & 20 & $3.0(1.1-4.9)$ & \\
\hline SBRT+TACE & 46 & $12.0(8.9-15.1)$ & \\
\hline \multicolumn{3}{|l|}{ Radiation Dose } & 0.376 \\
\hline$\leq 27.5 \mathrm{~Gy}$ & 4 & $3.0(0-8.9)$ & \\
\hline 30-35Gy & 25 & $8.0(6.8-9.2)$ & \\
\hline$\geq 40 G y$ & 41 & $11.0(7.7-14.3)$ & \\
\hline \multicolumn{3}{|c|}{ Origination of PVTT } & 0.079 \\
\hline Right branch & 49 & $8.0(5.8-10.2)$ & \\
\hline Left branch & 21 & $13.0(8.5-17.5)$ & \\
\hline \multicolumn{3}{|c|}{ Type of tumor thrombosis } & 0.107 \\
\hline Type ॥ & 42 & $8.0(5.5-10.5)$ & \\
\hline Type III & 27 & $14.0(8.6-19.4)$ & \\
\hline \multicolumn{3}{|c|}{ Response (3 m after SBRT) } & 0.000 \\
\hline$C R$ & 6 & $*$ & \\
\hline$P R$ & 43 & $13.0(10.0-16.0)$ & \\
\hline SD & 4 & $* *$ & \\
\hline PD & 9 & $4.0(1.1-6.9)$ & \\
\hline
\end{tabular}

Abbreviations: SBRT Stereotactic body radiotherapy, AFP Alpha-fetoprotein, $R T$ Radiotherapy, $C R$ Complete response, $P R$ Partial response, $S D$ Stable disease, $P D$ Progressive disease. * The medium survival for patients with CR was not reported because only two patients died in 7 th and 11th month. The other four patients were alive, 1 patient with 15 months follow-up, 3 patients with 16 months follow-up after SBRT. ** In the four patients with SD, 3 patients died in the 8th, 8th and 11th month, the other patient was alive with 12 months follow-up

comparable. With a median survival time of 12 months, our patients who got the chance to receive TACE after SBRT had longer survival time that is comparable to the results of the previous reports [27]. It is remarkable that patients in the present study were originally considered to harbor unresectable tumor or had contraindication for TACE. This subgroup of patients was expected to have lower OS rate. In view of such adverse baseline characteristics, the therapeutic outcome with median OS of 10 months seems satisfactory.

For the 5 patients who achieved near CR within the first few weeks, all were alive at the last follow-up times of $20,17,17,16$, and 10 months respectively. Those had $\mathrm{CR}$ at 3 months after SBRT also showed longer OS. Patients with good PVTT response to SBRT usually have better survival. As for the radiation dose, patients receiving $40 \mathrm{~Gy}$ or more seems to have longer OS (Table 3). Hence, We suggest higher radiation dose ( $\geq 40$ Gy in 5 fractions) for the SBRT treatment of PVTT, with the aim of achieving adequate therapeutic response and better survival.

The natural history of HCC patients with PVTT dictates only 2.4-4 months of survival duration [8]. Although Sorafenib provided a significant survival benefit in randomized phase III trials, the gain was modest (23 months) [29, 30]. Radiotherapy may improve survival chance for such patients. Yeh et al. reported a large series of HCC with PVTT treated by conventional three-dimensional conformal radiotherapy (3D-CRT), but the median survival time was only 7.0 months [31]. Matsuo et al. compared the efficacy of SBRT with 3D-CRT in the management of PVTT and better therapeutic results for the former was observed, with 1-year OS rates of $49.3 \%$ in SBRT group vs. $29.3 \%$ in 3D-CRT group. Taking all the results into consideration, SBRT seems to be of therapeutic benefit for HCC patients with PVTT, especially for those who had contraindication for TACE or surgery.

SBRT was well tolerated in our patient series. Only mild radiation acute side effects were observed in some of cases. No treatment-related deaths or serious adverse events were encountered. Furthermore, no radiation induced liver disease was observed, even in those receiving TACE after SBRT. Those who experienced deterioration of liver function were mainly due to intrahepatic tumor progression. The low toxicity of SBRT in the management of PVTT was in line with the results of other SBRT studies [26, 27].

There are several limitations of the present study that should be addressed. First, only contrast enhanced CT and/or MRI were used for the assessment of PVTT response, at 1,3 , and then every 3 months after SBRT. If liver vessel Doppler scan had been utilized in this study, the portal vein blood flow changes after SBRT might have been detected with higher frequency. Since Doppler exams could be repeated in shorter intervals, more information could have been collected in this respect. Second, the study was retrospective, with widely varied therapeutic modalities after SBRT for this group of patients, including TACE, surgical resection, and one liver transplantation. In addition, some patients received different number of TACE sessions. These could represent sources of bias for the observed data, and one should be cautious in the interpretation of the clinical outcomes. A well designed prospective study is warranted to validate the results. 


\section{Conclusions}

SBRT can be used as the first-line therapy for HCC patients with extensive PVTT originally considered unsuitable for surgical resection or TACE. The dynamic change of tumor thrombosis in time after SBRT has been described. Thrombus shrinkage and portal vein flow restoration can be achieved in the majority of cases. Thereafter, SBRT may enable patients to pursue consolidative local treatments, such as surgery and TACE procedure. Such therapeutic strategy may result in improved survival benefit, especially for those who do receive further therapies after SBRT.

\begin{abstract}
Abbreviations
3D-CRT: Three-dimensional conformal radiotherapy; AFP: Alpha-fetoprotein; CTCAE: Common Terminology Criteria for Adverse Events; D1cc: Maximal dose to 1 cC; HCC: Hepatocellular carcinoma; LC: Local control; mRECIST: Modified Response Evaluation Criteria in Solid Tumors; OARs: Organs at risk; OS: Overall survival; PD: Progressive disease; PR: Partial response; PVTT: Portal vein tumor thrombus; SBRT: Stereotactic body radiotherapy; SD: Stable disease; TACE: Transarterial chemoembolization
\end{abstract}

\section{Acknowledgements}

The authors would like to thank Professor Steve P. Lee from Department of Radiation Oncology, UCLA for editorial assistance.

\section{Funding}

This study was supported by the National Natural Science Foundation of China (Grant No. 81572952).

\section{Availability of data and materials}

The datasets used and analyzed during the current study are available from the corresponding author on reasonable request.

\section{Authors' contributions}

YJS participated in the design of the study and wrote the manuscript; WY and JX carried out the clinical data analysis and participated in writing of the manuscript; $X Q R, Y L G$, and BCZ interpreted the clinical data; TM and JJW contribute with the clinical data of TACE; QHL and QGH read and interpreted the $C T$ and MRI image. $L S, X L B$ and TBL contribute with the clinical data of surgery and radiotherapy; QCW conceived the study and wrote the manuscript. All authors read and approved the final manuscript.

\section{Ethics approval and consent to participate}

This study was approved by the Ethics Committee of SAHZU, and was carried out in accordance with the Declaration of Helsinki.

\section{Consent for publication}

Not applicable.

\section{Competing interests}

The authors declare that they have no competing interests.

\section{Publisher's note}

Springer Nature remains neutral with regard to jurisdictional claims in published maps and institutional affiliations.

\section{Author details}

'Department of Radiation Oncology, The Second Affiliated Hospital, Zhejiang University School of Medicine, Jiefang Road 88, Hangzhou 310009, People's Republic of China. ${ }^{2}$ Department of Hepatobiliary and Pancreatic Surgery, Zhejiang Provincial Key Laboratory of Pancreatic Disease, The Second Affiliated Hospital, Zhejiang University School of Medicine, Hangzhou 310009, People's Republic of China. ${ }^{3}$ Department of Radiology, The Second Affiliated Hospital, Zhejiang University School of Medicine, Hangzhou 310009, People's Republic of China. ${ }^{4}$ Ministry of Education Key Laboratory of Cancer
Prevention and Intervention, Zhejiang University Cancer Institute, Hangzhou 310009, People's Republic of China.

Received: 29 May 2018 Accepted: 20 September 2018

Published online: 25 September 2018

\section{References}

1. Siegel RL, Miller KD, Jemal A. Cancer Statistics, 2017. CA Cancer J Clin. 2017; 67(1):7-30.

2. Chen W, Zheng R, Baade PD, et al. Cancer statistics in China, 2015. CA Cancer J Clin. 2016;66(2):115-32.

3. Roayaie S, Blume IN, Thung SN, et al. A system of classifying microvascular invasion to predict outcome after resection in patients with hepatocellular carcinoma. Gastroenterology. 2009;137(3):850-5.

4. Yuan BH, Yuan WP, Li RH, et al. Propensity score-based comparison of hepatic resection and transarterial chemoembolization for patients with advanced hepatocellular carcinoma. Tumour Biol. 2016;37(2):2435-41.

5. Zhang ZM, Lai EC, Zhang C, et al. The strategies for treating primary hepatocellular carcinoma with portal vein tumor thrombus. Int J Surg. 2015; 20:8-16.

6. Chan SL, Chong CC, Chan AW, Poon DM, Chok KS. Management of hepatocellular carcinoma with portal vein tumor thrombosis: Review and update at 2016. World J Gastroenterol. 2016;22(32):7289-300.

7. Zhong JH, Peng NF, You XM, et al. Tumor stage and primary treatment of hepatocellular carcinoma at a large tertiary hospital in China: A real-world study. Oncotarget. 2017;8(11):18296-302.

8. Schöniger-Hekele M, Müller C, Kutilek M, Oesterreicher C, Ferenci P, Gangl A. Hepatocellular carcinoma in Central Europe: prognostic features and survival. Gut. 2001;48(1):103-9.

9. Li SH, Wei W, Guo RP, et al. Long-term outcomes after curative resection for patients with macroscopically solitary hepatocellular carcinoma without macrovascular invasion and an analysis of prognostic factors. Med Oncol. 2013;30(4):696.

10. Li SH, Guo ZX, Xiao CZ, et al. Risk factors for early and late intrahepatic recurrence in patients with single hepatocellular carcinoma without macrovascular invasion after curative resection. Asian Pac J Cancer Prev. 2013;14(8):4759-63.

11. Bruix J, Sherman M. Management of hepatocellular carcinoma: an update. Hepatology. 2011;53(3):1020-2.

12. Cheng S, Yang J, Shen F, et al. Multidisciplinary management of hepatocellular carcinoma with portal vein tumor thrombus - Eastern Hepatobiliary Surgical Hospital consensus statement. Oncotarget. 2016;7(26): 40816-29.

13. Kokudo T, Hasegawa K, Matsuyama Y, et al. Survival benefit of liver resection for hepatocellular carcinoma associated with portal vein invasion. J Hepatol. 2016;65(5):938-43.

14. Chen XP, Qiu FZ, Wu ZD, et al. Effects of location and extension of portal vein tumor thrombus on long-term outcomes of surgical treatment for hepatocellular carcinoma. Ann Surg Oncol. 2006;13(7):940-6.

15. Peng ZW, Guo RP, Zhang YJ, Lin XJ, Chen MS, Lau WY. Hepatic resection versus transcatheter arterial chemoembolization for the treatment of hepatocellular carcinoma with portal vein tumor thrombus. Cancer. 2012; 118(19):4725-36.

16. Chok KS, Cheung TT, Chan SC, Poon RT, Fan ST, Lo CM. Surgical outcomes in hepatocellular carcinoma patients with portal vein tumor thrombosis. World J Surg. 2014;38(2):490-6.

17. Katagiri S, Yamamoto M. Multidisciplinary treatments for hepatocellular carcinoma with major portal vein tumor thrombus. Surg Today. 2014;44(2): 219-26.

18. Omata M, Lesmana LA, Tateishi R, et al. Asian Pacific Association for the Study of the Liver consensus recommendations on hepatocellular carcinoma. Hepatol Int. 2010;4(2):439-74.

19. Kudo M, Matsui O, Izumi N, et al. JSH consensus-based clinical practice guidelines for the management of hepatocellular carcinoma: 2014 update by the liver cancer study group of Japan. Liver Cancer. 2014;3(3-4):458-68.

20. Yu JI, Park HC. Radiotherapy as valid modality for hepatocellular carcinoma with portal vein tumor thrombosis. World J Gastroenterol. 2016;22(30):6851-63.

21. Matsuo Y, Yoshida K, Nishimura H, et al. Efficacy of stereotactic body radiotherapy for hepatocellular carcinoma with portal vein tumor thrombosis/inferior vena cava tumor thrombosis: evaluation by comparison 
with conventional three-dimensional conformal radiotherapy. J Radiat Res. 2016;57(5):512-23.

22. Xi M, Zhang L, Zhao L, et al. Effectiveness of stereotactic body radiotherapy for hepatocellular carcinoma with portal vein and/or inferior vena cava tumor thrombosis. PLoS One. 2013;8(5):e63864.

23. Bruix J, Sherman M. Management of hepatocellular carcinoma. Hepatology. 2005;42(5):1208-36.

24. Shuqun C, Mengchao W, Han C, et al. Tumor thrombus types influence the prognosis of hepatocellular carcinoma with the tumor thrombi in the portal vein. Hepatogastroenterology. 2007;54(74):499-502.

25. Lencioni R, Llovet JM. Modified RECIST (mRECIST) assessment for hepatocellular carcinoma. Semin Liver Dis. 2010;30(1):52-60.

26. Lee SU, Park JW, Kim TH, et al. Effectiveness and safety of proton beam therapy for advanced hepatocellular carcinoma with portal vein tumor thrombosis. Strahlenther Onkol. 2014;190(9):806-14.

27. Kang J, Nie Q, Du R, et al. Stereotactic body radiotherapy combined with transarterial chemoembolization for hepatocellular carcinoma with portal vein tumor thrombosis. Mol Clin Oncol. 2014;2(1):43-50.

28. Bujold A, Massey CA, Kim JJ, et al. Sequential phase I and II trials of stereotactic body radiotherapy for locally advanced hepatocellular carcinoma. J Clin Oncol. 2013;31(13):1631-9.

29. Llovet JM, Ricci S, Mazzaferro V, et al. Sorafenib in advanced hepatocellular carcinoma. N Engl J Med. 2008;359(4):378-90.

30. Cheng AL, Kang YK, Chen Z, et al. Efficacy and safety of sorafenib in patients in the Asia-Pacific region with advanced hepatocellular carcinoma: a phase III randomised, double-blind, placebo-controlled trial. Lancet Oncol. 2009;10(1):25-34.

31. Yeh SA, Chen YS, Perng DS. The role of radiotherapy in the treatment of hepatocellular carcinoma with portal vein tumor thrombus. J Radiat Res. 2015;56(2):325-31.

Ready to submit your research? Choose BMC and benefit from:

- fast, convenient online submission

- thorough peer review by experienced researchers in your field

- rapid publication on acceptance

- support for research data, including large and complex data types

- gold Open Access which fosters wider collaboration and increased citations

- maximum visibility for your research: over $100 \mathrm{M}$ website views per year

At $\mathrm{BMC}$, research is always in progress.

Learn more biomedcentral.com/submissions 